Abstract

\title{
Entropic Dynamics on Gibbs Statistical Manifolds ${ }^{\dagger}$
}

\section{Pedro Pessoa, Felipe Xavier Costa and Ariel Caticha}

Department of Physics, University at Albany (SUNY), Albany, NY, USA

† Presented at the Entropy 2021: The Scientific Tool of the 21st Century, 5-7 May 2021; Available online: https://sciforum.net/conference/Entropy2021/.

Published: 5 May 2021

In a modern approach to statistical physics [Jaynes: Phys. Rev. 106, 620, 1957], Gibbs distributions appear naturally as a solution to a well set optimization problem: maximizing entropy under a set of expected values constraints. Generally in physics, the space in which a canonical distribution is defined are the microstates and the set expected values define the macrostates, which can be used as coordinates in a space of such canonical distributions (statistical manifold). In the field of Information Geometry [Amari and Nagaoka: Methods of Information Geometry, American Mathematical Soc., 2007] [Ruppeiner: Rev. Mod. Phys. 68, 313, 1996] these distributions happen to have deeply interesting geometrical properties such as their metric tensor is a covariance matrix and important thermodynamical objects, such as free energy, appear naturally. This work aims to provide a systematic way to create dynamical systems in a space of canonical distributions. These dynamics are derived as an application of entropic methods of inference i.e., that is a form of entropic dynamics [Caticha: Entropy 17, 6110, 2015]. As an interesting result, the average motion in such a dynamical process reduces to the Onsager Relations [Onsager: Phys. Rev. 37, 405, 1931], derived from purely probabilistic - not intrinsically thermodynamical-arguments. This can give new insight on fields such as critical phenomena and renormalization groups as well as deal with statistical problems in which the microstate dynamics is not so well defined such as economics and ecology.

(C) 2021 by the authors. Licensee MDPI, Basel, Switzerland. This article is an open access article distributed under the terms and conditions of the Creative Commons Attribution (CC BY) license (http://creativecommons.org/licenses/by/4.0/). 